\title{
Effectiveness of Pharmacist-convened Multidisciplinary Clinical Team Meetings in Promoting Appropriate Benzodiazepine Receptor Agonist Use
}

\author{
Mayumi Geka, ${ }^{a}$ Ken Inada, ${ }^{*}, b$ Satoru Shimizu, ${ }^{c}$ Yujiro Geka, ${ }^{a}$ Yuka Takahashi, ${ }^{a}$ \\ Yukihiro Hamada, ${ }^{a}$ Katsuji Nishimura, ${ }^{b}$ and Toshimi Kimura ${ }^{a}$ \\ ${ }^{a}$ Department of Pharmacy, Tokyo Women's Medical University Hospital; 8-1 Kawada-cho, Shinjuku-ku, \\ Tokyo 162-8666, Japan: ${ }^{b}$ Department of Psychiatry, School of Medicine, Tokyo Women's Medical University; \\ 8-1 Kawada-cho, Shinjuku-ku, Tokyo 162-8666, Japan: and 'Department of Medical Education, School of \\ Medicine, Tokyo Women's Medical University; 8-1 Kawada-cho, Shinjuku-ku, Tokyo 162-8666, Japan.
}

(Received October 17, 2018; Accepted February 14, 2019)

\begin{abstract}
Benzodiazepine receptor agonists (BZRAs) are used in the treatment of a wide variety of clinical conditions. Although clinical practice guidelines discourage high dosage or long-term use of BZRAs, they are prescribed in clinical settings. This study aimed to investigate whether the pharmacists at multidisciplinary clinical team meetings can help reduce BZRA use and promote appropriate use of these drugs. The psychiatric unit of the Tokyo Women's Medical University Hospital occupies two floors, with 31 beds on Floor A and 34 beds on Floor B. The multidisciplinary clinical team meetings were held once a week in each ward. During the meetings, the pharmacists comprehensively assessed the number of BZRA doses administered and the equivalent diazepam doses, presented their prescription recommendations aimed at dosage reduction, and shared their views with the entire clinical team. This intervention was commenced on Floor A in 2014 and on Floor B in 2015. The average number of BZRAs in each period and equivalent diazepam doses were assessed for 273 psychiatric inpatients hospitalized from April to June in 2013, 2014, and 2015. Changes in the number of BZRA doses administered were assessed per floor per year. The results showed a statistically significant decrease between years with and without interventions. The intervention of pharmacists allowed multidisciplinary clinical team members to gain the same understanding about BZRA use and formulation of drug therapy plans. The results suggest that the intervention of pharmacists at clinical team meetings can strategically lead to decreased BZRA dosages and their proper use.
\end{abstract}

Key words — pharmacist; multidisciplinary team; benzodiazepine; proper use

\section{INTRODUCTION}

Owing to their sedative, hypnotic, anxiolytic, anticonvulsant, muscle relaxant, and other effects, benzodiazepine receptor agonists (BZRAs) are used to treat a wide variety of conditions such as anxiety, insomnia, seizures, and convulsions. ${ }^{1-3)}$ However, the use of BZRAs might result in adverse effects, e.g., daytime drowsiness, dizziness, ataxia, psychomotor disturbance, anterograde amnesia, and potential dependence..$^{4-6)}$ Consequently, expert consensus and prescription guidelines for BZRAs discourage polypharmacy or long-term use. ${ }^{7,8)}$ Nevertheless, BZRA use remains extremely high in Japan compared with that in other countries. ${ }^{9)}$ Various strategies have been suggested to prevent BZRA polypharmacy. Thus, medical fee reimbursement policies might restrict the prescribed dosage to a 30-d maximum or reduce reimbursements if three or more types of anxiolytic or

\footnotetext{
*e-mail: inada.ken@twmu.ac.jp
}

hypnotic drugs are used. ${ }^{5,6)}$ However, a study of psychiatric outpatients has reported limited and lowerthan-anticipated success for such strategies. ${ }^{10)}$ Further, even after their implementation, concurrent use of two or more types of BZRA drugs has been reported to still exceed $32 \%$. Hence, psychiatrists need to take stock of these problems, reduce BZRA dosages in patients on polypharmacy, and promote appropriate use of these drugs.

Pharmacists at the psychiatric wards of the Tokyo Women's Medical University Hospital (hereinafter referred to as "the hospital") have been assessing BZRA use based on specific clinical criteria (Table 1) and have been offering prescription recommendations to attending psychiatrists. By recording their assessments, recommendations, and instructions for patients in medical charts, the pharmacists aimed to collectively share the information with multidisciplinary clinical teams, e.g., nurses and therapists. However, because of the highly specialized nature of the written information, everyone on the team could 
Table 1. Pharmacist's Assessment Criteria for Prescription Recommendations

Benzodiazepine dosage: 3 or more benzodiazepines AND/ OR $20 \mathrm{mg}$ or more of the diazepam equivalent

Patient's night-time sleeping patterns

Daytime activity patterns

Main pharmacotherapy (antipsychotic and antidepressant) adjustments

Duration of benzodiazepine use

not easily acquire a shared understanding of the information. Further, there are no clearly established standards for continuing or discontinuing BZRA administration. Therefore, various members of a team tended to offer different recommendations to psychiatrists, which made it difficult for the psychiatrists to define policies for BZRA administration. Since the appropriate BZRA use was obstructed by their action, the pharmacists realized the need for a more effective information-sharing strategy to raise awareness, reinforce the goal of reducing BZRA dosages, and promote the appropriate use of these drugs. With this objective in mind, the pharmacists decided to convene multidisciplinary clinical team meetings where various members of medical staff could exchange views.

This study aimed to determine whether information sharing during these team meetings led to a reduction of BZRA dosage and promote appropriate BZRA use. Changes in the status of BZRA use in the hospital's psychiatric wards before and after the intervention were investigated retrospectively from medical charts.

\section{METHODS}

\section{Inauguration of Multidisciplinary Clinical Team} Meetings by Pharmacists The psychiatric unit of the Tokyo Women's Medical University Hospital occupies two floors, with 31 beds on Floor A and 34 beds on Floor B. Sharing the same layout and treating patients with similar afflictions, both are critical care wards for inpatients, with the average hospitalization period of $60 \mathrm{~d}$. The psychiatrists, pharmacists, and other medical staff are different on each floor. The multidisciplinary clinical team meetings were held once a week in each ward. The team consisted of psychiatrists, pharmacists, nurses, therapists, psychologists, and social workers.

The pharmacists played the central role in compre- hensively assessing the BZRA dosage. At these team meetings, the pharmacists screened out patients, who, based on Table 1, presented their prescription recommendations aimed at dosage reduction, and made time for explaining how to assess BZRAs. Thus, the pharmacists continued to provide the team opportunities for considering appropriate BZRA use. If patients taking the same BZRA dosage were present before the next conference, the pharmacists announced it again. This intervention was commenced on Floor A in 2014 and on Floor B in 2015.

Subjects and Study Design Initially, the subjects included 366 patients confined in the hospital's psychiatric wards from April to June of 2013, 2014, and 2015. Patients were diagnosed using the International Statistical Classification of Diseases and Related Health Problems 10 (ICD 10). The exclusion criteria were as follows: history of epilepsy and/or sleep apnea (19 patients), receiving electroconvulsive therapy during hospitalization (14 patients), not taking BZRAs during the study (39 patients), and hospitalization for more than once during the study period (20 patients); thus, 273 subjects were eventually included in the study. The study was conducted as a retrospective survey of their medical charts.

Assessment of the Effectiveness of Pharmacists' Intervention at Multidisciplinary Clinical Team Meetings

Changes in the number of BZRA doses administered Changes in the number of BZRA doses administered were assessed per floor per year. During the 3-month study period, from April to June, in each of the 3 years, the average daily BZRA dosage was analyzed in terms of the number of doses administered and the equivalent diazepam doses. Considering that some of medical staff who are medical interns change every 3 months, the study period was 3 months. Since the BZRA dosage varied for a patient during the initial hospitalization period and assessing the effectiveness of team meetings on BZRA dosage reduction at specific points in time was difficult, the average dosage administered per patient during the study period was assessed. Single-dose prescriptions were excluded from the average dosage.

Discontinuation of BZRA administration during the study period The number of patients who could tolerate the discontinuation of BZRA administration was determined.

Statistical analysis Comparison of the dosages 
Table 2. Patient Characteristics

\begin{tabular}{|c|c|c|c|c|c|c|}
\hline Year & \multicolumn{2}{|c|}{2013} & \multicolumn{2}{|c|}{2014} & \multicolumn{2}{|c|}{2015} \\
\hline Floor & A & $\mathrm{B}$ & A & B & A & $\mathrm{B}$ \\
\hline Intervention $^{\mathrm{a}}$ & - & - & + & - & + & + \\
\hline$n$ & 30 & 47 & 44 & 57 & 36 & 59 \\
\hline Gender (male/female) & $7 / 23$ & $13 / 34$ & $16 / 28$ & $21 / 36$ & $8 / 28$ & $14 / 45$ \\
\hline $\mathrm{Age}^{\mathrm{b}}$ & $49.5(24-80)$ & $48(19-87)$ & $48.5(14-84)$ & $49(16-89)$ & $44(16-75)$ & $47(17-96)$ \\
\hline \multicolumn{7}{|l|}{ Disease } \\
\hline Organic, including symptomatic, mental disorders & 4 & 1 & 1 & 2 & 1 & 2 \\
\hline $\begin{array}{l}\text { Mental and behavioral disorders due to psychoactive } \\
\text { substance use }\end{array}$ & 2 & 2 & 0 & 4 & 2 & 2 \\
\hline Schizophrenia, schizotypal, and delusional disorders & 9 & 21 & 15 & 13 & 11 & 16 \\
\hline Mood (affective) disorders & 10 & 15 & 23 & 29 & 13 & 30 \\
\hline Neurotic, stress-related, and somatoform disorders & 4 & 6 & 4 & 5 & 6 & 6 \\
\hline $\begin{array}{l}\text { Behavioral syndromes associated with physiological } \\
\text { disturbances and physical factors }\end{array}$ & 1 & 1 & 0 & 2 & 0 & 2 \\
\hline Disorders of adult personality and behavior & 0 & 1 & 0 & 0 & 0 & 0 \\
\hline Mental retardation & 0 & 0 & 0 & 0 & 0 & 0 \\
\hline Disorders of psychological development & 0 & 0 & 1 & 2 & 2 & 1 \\
\hline $\begin{array}{l}\text { Behavioral and emotional disorders with onset usually } \\
\text { occurring in childhood and adolescence }\end{array}$ & 0 & 0 & 0 & 0 & 1 & 0 \\
\hline Unspecified mental disorder & 0 & 0 & 0 & 0 & 0 & 0 \\
\hline
\end{tabular}

${ }^{\mathrm{a}}$ Intervention; +, Non-Intervention: - ${ }^{\mathrm{b}}$ Median (min-max) .

used on Floors A and B in 2013, 2014, and 2015 involved different patients each year. The team meetings were held at different times on Floors A and B. Further, since the distinct focus of this study was on pre- and post-meeting dosages, a longitudinal comparison of dosage changes was necessary.

The longitudinal changes in dosages were compared using analysis of variance. Statistical analysis was performed using the repeated measures method and GLM procedure in SAS version 9.4 (SAS Institute, Cary). The level of significance was set at $p<$ 0.05 .

Ethics This study was performed in conformity with the Declaration of Helsinki and ethical policies for clinical research and was approved by the Ethics Committee of the Tokyo Women's Medical University Hospital (No. 3301).

\section{RESULTS}

Patient Characteristics The characteristics of the 273 subjects, classified according to floor and year of study, are shown in Table 2.

Assessment of the Effectiveness of Pharmacists' Intervention at Multidisciplinary Clinical Team Meetings

Changes in average BZRA dosage Factoring in their time dependence, the average numbers of BZRA administered on Floor A in 2013, 2014, and 2015 were subjected to analysis of variance. The results showed a statistically significant difference between 2013 when there was no intervention and 2014 when there was an intervention. No statistically significant difference was noted between 2014 and 2015, both of which involved interventions (Fig. 1).

Factoring in their time dependence, the average numbers of BZRA administered on Floor B in 2013, 2014 , and 2015 were also subjected to analysis of variance. The results showed no statistically significant difference between 2013 and 2014, neither of which involved an intervention. However, statistically significant difference was noted between each of these years and 2015, during which an intervention occurred (Fig. 2).

Comparison of the average equivalent diazepam doses showed similar results (Figs. 3 and 4).

Discontinuation of BZRA use during the study period The ratios of the subjects who could discontinue BZRA use on each floor in 2013, 2014, and 2015 were as follows: Floor A: 2/30(6.7\%), 21/44 (48\%), and 17/36(47\%); Floor B: $4 / 47(8.5 \%), 2 /$ $57(3.5 \%)$, and $16 / 59(27 \%)$, respectively. 


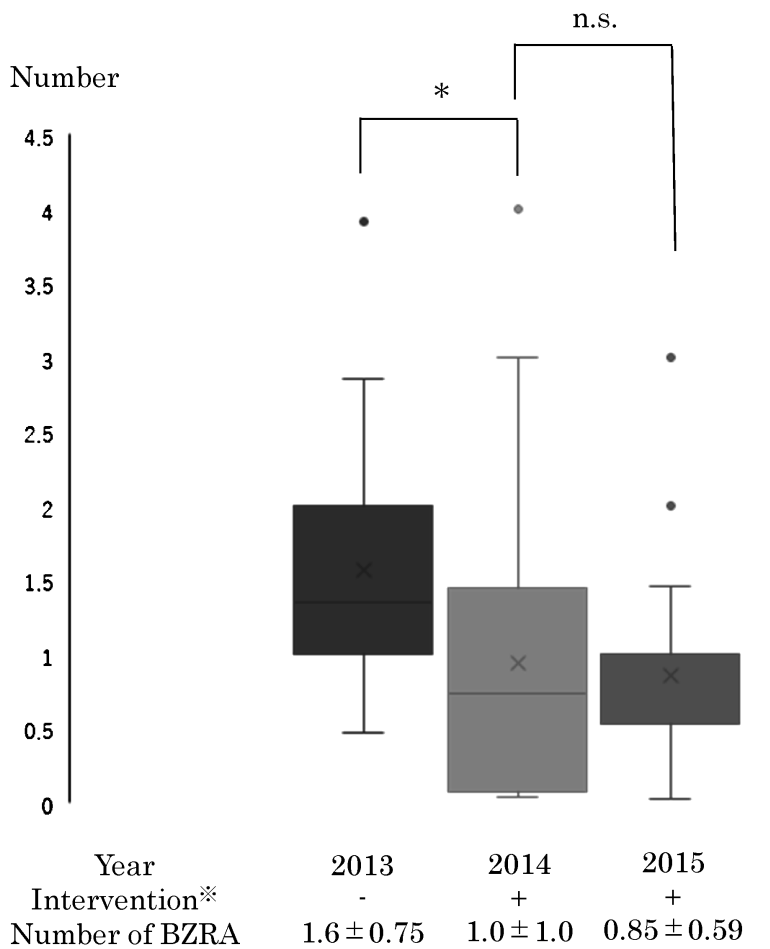

Fig. 1. Average Numbers of Pre- and Post-intervention BZRA Doses on Floor A

*Intervention : + , Non-Intervention : - . ${ }^{*} p<0.05$, n.s : not significant.

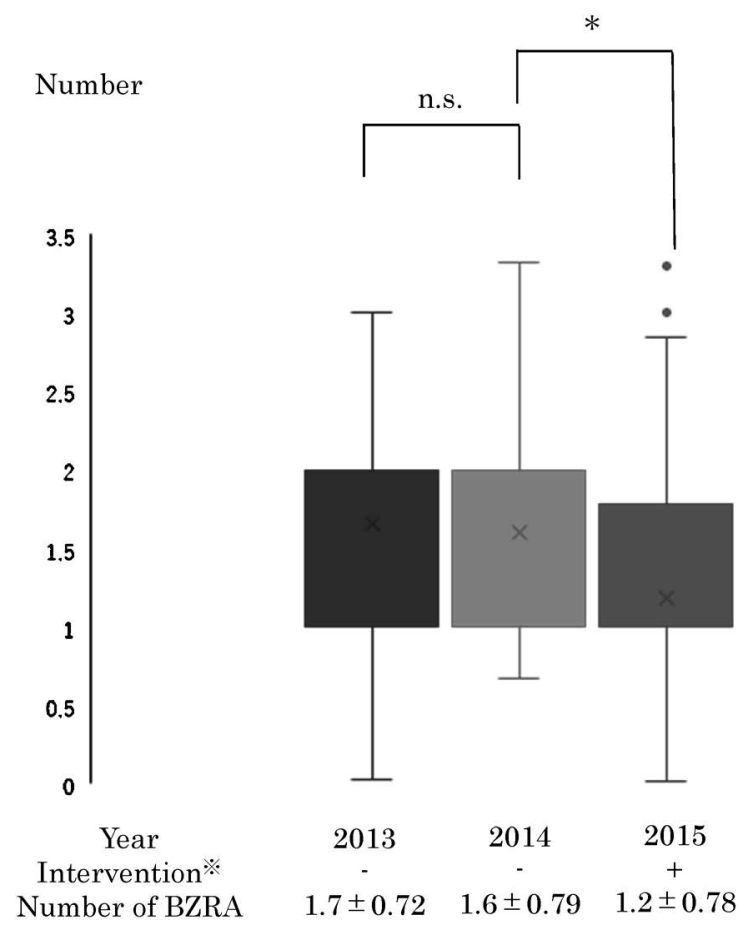

Fig. 2. Average Numbers of Pre- and Post-intervention BZRA doses on Floor B

*Intervention : + , Non-Intervention : - . ${ }^{*} p<0.05$, n.s : not significant.

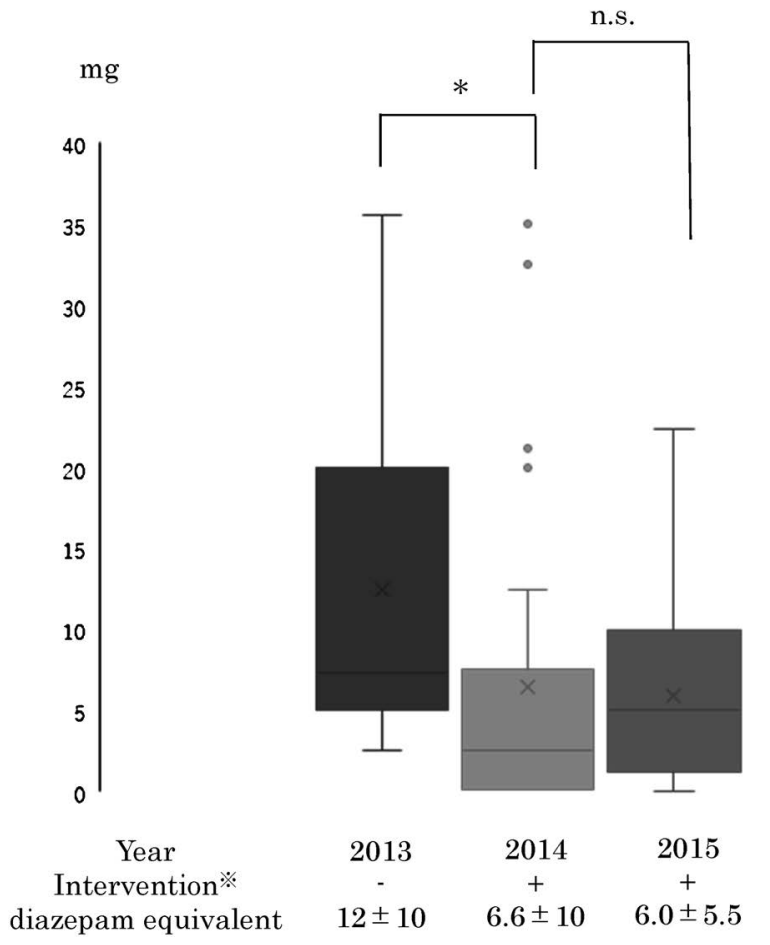

Fig. 3. Average Pre- and Post-intervention Diazepam Equivalent BZRA Doses (mg) on Floor A

*Intervention : + , Non-Intervention : $-.{ }^{*} p<0.05$, n.s : not significant.

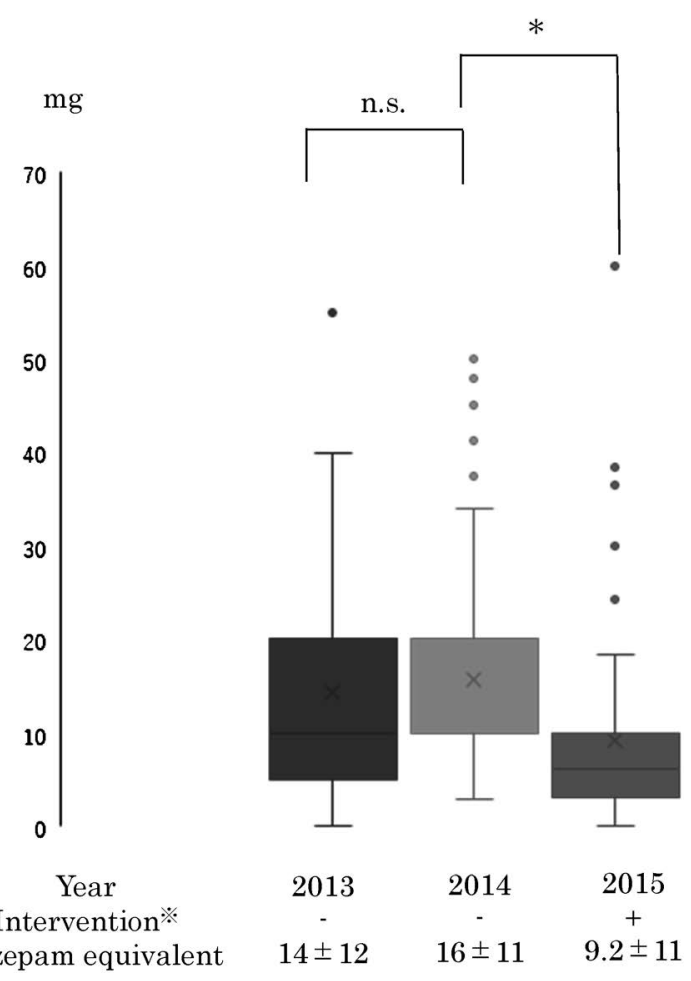

Fig. 4. Average Pre- and Post-intervention Diazepam Equivalent BZRA Doses (mg) on Floor B

*Intervention : + , Non-Intervention : $-.{ }^{*} p<0.05$, n.s : not significant. 


\section{DISCUSSION}

BZRA polypharmacy and long-term use are pressing problems in Japan. At the psychiatric wards of the Tokyo Women's University Hospital, clinical pharmacists have been offering individualized prescription recommendations to attending psychiatrists, under the supervision of psychiatricspecialist pharmacists, to promote appropriate BZRA use. The average numbers of BZRA doses administered according to the individualized recommendations were as follows: Floor A in 2013: 1.6 \pm 0.75 ; Floor B in 2013: $1.7 \pm 0.72$; and Floor B in 2014: 1.6 \pm 0.79 (Figs. 1 and 2, Non-Intervention). The average equivalent diazepam doses were as follows: Floor A in 2013: $12 \pm 10 \mathrm{mg}$; Floor B in 2013: $14 \pm 12$ $\mathrm{mg}$; and Floor B in 2014: 16 $\pm 11 \mathrm{mg}$ (Figs. 3 and 4, Non-Intervention). In many cases, these doses were within the dosage range indicated in the package insert, which might be considered a result of the pharmacists' proactive recommendations to the attending psychiatrists. However, since polypharmacy of BZRA drugs, discouraged by the guidelines, still persisted, there was a need for dosage reduction or monotherapy.

In hospital settings, BZRA dosage reduction is hindered by differences in the perception and priorities and the lack of a meeting of minds among medical staff. For instance, even if pharmacists comprehensively assess BZRA use from a pharmacological perspective and propose dosage reduction, other medical staff often prioritize the alleviation of minor anxiety or insomnia and favor an increased dosage. Moreover, even if individualized prescription recommendations are formulated, not everyone on the clinical team might share the same level of comprehension because of a highly specialized nature of the information. To overcome this problem, pharmacists personally explained how to assess the BZRA dosage and stressed on understanding the necessity for decreasing polypharmacy.

To foster a collective understanding and ownership of the dosage reduction issue among various health professionals involved, the pharmacists convened multidisciplinary clinical team meetings. At these meetings, psychiatrists established their strategies for future dosage adjustments by using prescription recommendations for BZRA use based on comprehensive assessments by the pharmacists and shared them with the team members. A deeper comprehension of the drugs' effects and the importance of dosage reduction were fostered, aiding other health professionals in their nursing, therapeutic, and psychological programs for the patients.

Comparison of BZRA dosages on each floor during each year of the study period was time-dependent in this study. At contiguous points in time, significant differences in dosages were noted before and after the intervention on both floors (Figs. 1-4). Thus, on Floor A, where the intervention started in 2014, the dosage amounts significantly decreased from the preintervention levels, and smaller doses were sustained thereafter (Figs. 1 and 3). Similarly, on Floor B, where the intervention was commenced in 2015 , the dosage significantly decreased after the intervention (Figs. 2 and 4). The intervention represented by these multidisciplinary clinical team meetings, convened by the pharmacists, might be considered one of the contributing factors to this decrease.

Statistically significant differences were noted between the dosage amounts on Floors A and B before and after the team meetings were held. However, although different subjects were included each year, the data for the consecutive years of 2013, 2014, and 2015 had distinctive characteristics. Hence, the data for each year needed to be analyzed longitudinally. This study might be believed to have employed the most appropriate statistical method for analyzing longitudinal changes.

According to Furukawa's systematic review, ${ }^{11)}$ the concurrent use of BZRAs during the initial stage of pharmacotherapy increases the continuance rate of the therapy. However, in actual clinical practice, long-term BZRA use persists..$^{9,12,13)}$ In this view, success of this study in terms of increasing the ratio of patients who are able to discontinue post-intervention BZRA use shows a highly beneficial effect of the multidisciplinary clinical team meetings convened by pharmacist.

Thompson et al. ${ }^{14)}$ reported that the introduction of an alert system that goes off on a prescription screen whenever a psychiatrist prescribes concurrent antipsychotic drugs led to lower rates of polypharmacy; however, the success was limited. Hazra et al. ${ }^{15)}$ reported that when confronting the problem of antipsychotic polypharmacy, reiteration by pharmacists of the risks of polypharmacy and the importance of dosage reduction during weekly multidisciplinary 
meetings, in addition to individualized cautions directed to prescribing psychiatrists, increased monotherapy. These reports, as well as the results of this study, highlight the importance of reinvigorating the awareness, among not only the attending psychiatrists but also the entire clinical team, of the problem of polypharmacy and the need to promote the appropriate drug use.

By participating in the multidisciplinary clinical team meetings, the members of the team were able to ascertain the current status of BZRA use. As a strategy for promoting appropriate BZRA use, the meetings can provide a useful setting where pharmacists can present their recommendations for prescriptions and dosages.

This study has the following limitations. First, the study was conducted at a single institution; thus, similar multi-institutional studies need to be performed to confirm the results. Second, not only decreasing of BZRA dosages but also of incidents is important; however, the rate of acceptance of recommendation by psychiatrists and the change in incident number could not be revealed, because this was a retrospective study. Third, clinical factors such as the duration of patients' illnesses could not be adequately incorporated into the analysis.

\section{CONCLUSION}

Multidisciplinary clinical team meetings where pharmacists can effectively share information on the current status of BZRA use and their prescription recommendations with other clinical team members can lead to reduced BZRA dosages.

Conflict of Interest This study was partly supported by an Intramural Research Grant (29-1) for Neurological and Psychiatric Disorders from the National Center of Neurology and Psychiatry and a Health and Labour Sciences Research Grant. Ken Inada received speaker's honoraria from Sumitomo Dainippon Pharma, Eisai, Eli Lilly, Janssen, Meiji Seika Pharma, Mochida, MSD, Novartis, Otsuka, Shionogi, Mitsubishi Tanabe Pharma, and Yoshitomi and research grants/support from MSD. Katsuiji Nishimura received speaker's honoraria from Meiji Seika Pharma, Mochida Pharmaceutical, Takeda Pharmaceutical, Eli Lilly, MSD, Shionogi, Janssen Pharmaceutical, Eisai, Otsuka Pharmaceutical, Novartis, Mitsubishi Tanabe Pharma, and Chugai
Pharmaceutical and research grants/support from Mochida Pharmaceutical, Takeda Pharmaceutical, Otsuka Pharmaceutical, Novartis, Mitsubishi Tanabe Pharma, Sumitomo Dainippon Pharma, MSD, Eisai, Tsumura, and Mebix. There are no conflict of interests for other authors.

\section{REFERENCES}

1) Charlson F., Degenhardt L., McLaren J., Hall W., Lynskey M., Pharmacoepidemiol. Drug Saf., 18, 93-103 (2009) .

2) Cloos J. M., Ferreira V., Curr. Opin. Psychiatry, 22, 90-95 (2009).

3) Fisher J., Sanyal C., Frail D., Sketris I., J. Clin. Pharm. Ther., 37, 7-21 (2012).

4) Hutchinson M. A., Smith P. F., Darlington C. L., Prog. Neurobiol., 49, 73-97 (1996).

5) Barker M. J., Greenwood K. M., Jackson M., Crowe S. F., Arch. Clin. Neuropsychol., 19, 437-454 (2004).

6) Holbrook A. M., Crowther R., Lotter A., Cheng C., King D., CMAJ, 162, 225-233 (2000) .

7) American Geriatrics Society 2015 Beers Criteria Update Expert Panel, J. Am. Geriatr. Soc., 63, 2227-2246 (2015).

8) Neutel C. I., Skurtveit S., Berg C., Sleep Med., 13, 893-897 (2012).

9) Takeshima N., Ogawa Y., Hayasaka Y., Furukawa T. A., Psychiatry Res., 237, 201207 (2016).

10) Okumura Y., Inada K., Matsumoto T., Shimizu S., Jpn. J. Clin. Psychopharmacol. 18, 1173-1188 (2015).

11) Furukawa T. A., Streiner D. L., Young L. T., Cochrane Database Syst. Rev., CD001026 (2002).

12) Vicens C., Bejarano F., Sempere E., Mateu C., Fiol F., Socias I., Aragonès E., Palop V., Beltran J. L., Piñol J. L., Lera G., Folch S., Mengual M., Basora J., Esteva M., Llobera J., Roca M., Gili M., Leiva A., Br. J. Psychiatry, 204, 471-479 (2014).

13) Veronese A., Garatti M., Cipriani A., Barbui C., Eur. J. Clin. Pharmacol., 63, 867-873 (2007).

14) Thompson A., Sullivan S. A., Barley M., Strange S. O., Moore L., Rogers P., Sipos A., Harrison G., Psychol. Med., 38, 705-715 
(2008).

15) Hazra M., Uchida H., Sproule B., Remington
G., Suzuki T., Mamo D. C., Psychiatry Clin. Neurosci., 65, 676-678 (2011). 